\title{
Comparing visual preferences between autism spectrum disorder (ASD) and normal children to explore the characteristics of visual preference of ASD children by improved visual preference paradigm: a case-control study
}

\author{
Xiaoyun Gong ${ }^{1}$, Xue $\mathrm{Li}^{1}$, Qiandong Wang ${ }^{2}$, Sio Pan Hoi ${ }^{3}$, Tingni Yin ${ }^{1}$, Liyang Zhao ${ }^{1}$, Fanchao Meng ${ }^{4}$, \\ Xuerong Luo ${ }^{4}$, Jing Liu ${ }^{1}$ \\ ${ }^{1}$ Peking University Sixth Hospital, Peking University Institute of Mental Health, NHC Key Laboratory of Mental Health, National Clinical \\ Research Center for Mental Disorders, Peking University, Peking University Sixth Hospital, Beijing, China; ${ }^{2}$ Beijing Key Laboratory of Applied \\ Experimental Psychology, National Demonstration Center for Experimental Psychology Education, Faculty of Psychology, Beijing Normal \\ University, Beijing, China; ${ }^{3}$ School of Psychological and Cognitive Science, Beijing Key Laboratory of Behavior and Mental Health, Peking \\ University, Beijing, China; ${ }^{4}$ Department of Psychiatry, The Second Xiangya Hospital, Central South University, Changsha, China \\ Contributions: (I) Conception and design: X Gong, X Li; (II) Administrative support: J Liu; (III) Provision of study materials or patients: X Li, J Liu; (IV) \\ Collection and assembly of data: X Gong; (V) Data analysis and interpretation: X Gong, Q Wang, SP Hoi; (VI) Manuscript writing: All authors; (VII) \\ Final approval of manuscript: All authors. \\ Correspondence to: Jing Liu. 51 Huayuanbei Road, Haidian District, Beijing, 100191 China. Email: ljyuch@bjmu.edu.cn; Xuerong Luo. Central South \\ University, Changsha, China. Email: luoxuerong@csu.edu.cn.
}

Background: One of features of Visual preference in autism spectrum disorder (ASD) is that they tend to social stimuli to nonsocial stimuli. Though it has been studied widely, until now the results are still not consistent. One of the most important reasons may be that the stimuli of the past visual preference paradigm have different movement patterns. Therefore, the present study aimed to improve the visual preference paradigm to reduce the bias caused by different movement of stimuli and explore the features of visual preference of ASD further.

Methods: Two hundred and seven children who met DSM-5 criteria for ASD and 125 typically developmental (TD) children were enrolled. They were matched in age and gender. Visual preference was measured by eye tracking technology. The subjects presented simultaneously with dynamic object images (DOI) and dynamic social images (DSI) with the same type and same speed of repetitive movement. Social interaction deficits of ASD were assessed by parents with Aberrant Behavior Checklist (ABC).

Results: Compared with TD children, the following findings were noted: (I) children with ASD paid less attention to social stimuli and more attention to nonsocial stimuli $(t=6.417, \mathrm{P}<0.001)$. (II) Increased preference to DSI was positively correlated with age $(\mathrm{R}=0.23, \mathrm{P}=0.001)$. (III) No association was noted between visual preference and IQ in children with ASD $(\mathrm{R}=0.081, \mathrm{P}=0.435)$. (IV) Reduced preference to DSI was negatively correlated with the severity of social interaction impairment rated by parents with $\mathrm{ABC}$ in children with ASD $(\mathrm{R}=0.237, \mathrm{P}=0.010)$.

Conclusions: Children with ASD exhibited abnormal visual preference for social and nonsocial stimuli.

Keywords: Autism spectrum disorder (ASD); eye tracking; visual preference; social interaction

Submitted Jun 28, 2021. Accepted for publication Jul 22, 2021.

doi: $10.21037 /$ tp-21-294

View this article at: https://dx.doi.org/10.21037/tp-21-294 


\section{Introduction}

The core manifestation of autism spectrum disorder (ASD) is social communication and interaction impairment (1), and abnormal social attention is an important symptom of social communication and interaction disorder in children with ASD. Therefore, a focus on features of visual preference for social and nonsocial stimuli in children with ASD can help to understand the key symptom of social interaction disorder. Meanwhile, the visual preference for social or nonsocial stimuli may provide the possibility to screen ASD with instrument measures.

The assessment of visual preference in ASD was mainly based on behavioral indicators, such as questionnaires or checklists including Communication and Symbolic Behavior Scales Developmental Profile (CSBS-DP), Modified Checklist for Autism Toddlers (M-CHAT), Autism Behavior Checklist and Social Responsiveness Scale (SRS) have been used widely across countries. These questionnaires or checklists have been proved to possess optimal or accepted reliability and validity and have played an important role in the evaluation of visual features in ASD. However, the results are influenced to a certain extent by several factors including the observation time and attention of the evaluator to children and the apprehension to items of scales of evaluator (2). Therefore, the results of scale assessment are relatively and inherently subjective and occasionally they are unavoidably causing unreliable screening results. In order to overcome various forms of bias, it is of great significance to explore more objective and simple methods that could result in measure of features of visual preference in ASD more effectively.

Eye movement technology is a remote table-mounted video-based eye-tracking system. It depends on the pupil/ corneal reflection and aims to estimate the point of gaze and record gaze behavior. Researchers can obtain all aspects of social attention indicators of ASD through eye tracking technology. It is objective, quantifiable, non-invasive, relatively inexpensive and easy to be implemented. It can also be applied to individuals of any age from infancy to adulthood or any functional level (3). The continuous development of eye movement technology in recent decades has enabled its wide use in the field of ASD research.

Currently, several studies have shown that the visual fixation characteristics of individuals with ASD are significantly different from those of normal developing individuals following processing of social stimuli in fixation time, fixation latency and fixation locus (4-6). For example, adolescents with ASD watching movies tend to spend significantly less fixation time on the people than on the background scene (7). Toddlers with ASD have a range of social visual attention deficits including reduced attention to others' faces $(5,8)$, eyes $(4,9-11)$, head regions (12) and biological motion (13), whereas their joint attention is poor $(14,15)$. Moreover, individuals with ASD display more visual preference to non-social objects $(4,16-18)$ than typical developing individuals, notably for some specific objects (e.g., trains, computers and geographic figures) and for parts of objects and repetitive movement (e.g., the rotating blades of a fan or the spinning of car wheels) $(19,20)$. Compared to other diagnostic groups, they also prefer to attend to highly repetitive images, such as repeating geometric shapes rather than social images $(3,16,17)$. Pierce et al. (17) examined simultaneously the eye gaze of subjects on videos of children doing yoga and found that children with ASD paid more attention to geometric pictures. Kou et al. (21) presented geometric figures of repetitive motion and videos of dancing children or adults to subjects at the same time and found that children with ASD preferred to look at geometric figures with repetitive motion compared to typically developmental (TD) children rather than looking at humans presented.

Although the eye movement technology has been used to explore the features of social attention of individuals with ASD and the detection of children with ASD, the majority of eye movement studies suggest that individuals with ASD have decreased visual attention to social stimuli compared to that of TD individuals. However, the results of previous studies are inconsistent. Sasson and Touchstone (18) used stimuli including pictures of a face paired with an object related or unrelated to restricted interests. A decreased fixation time on the social face was found only when the face was paired with objects related to restricted interest. Fischer et al. (22) used a "gap-overgap" paradigm to test social orientation by counting saccadic reaction time for peripheral targets, either a social or a non-social image. Both 9-year-old children with ASD and their controls demonstrated shorter saccadic reaction time when the peripheral target was a social image and no difference was noted between the two groups.

The results of previous studies are not totally consistent and certain differences are noted in the paradigm. Although the studies of Pierce et al. (17) and Kou et al. (21) had found that children with ASD preferred to look at geometric figures compared to typically developing children rather than looking at humans presented at the 
Table 1 Demographic and clinical characteristics for sample (mean \pm SD)

\begin{tabular}{lccc}
\hline Characteristics & ASD $(\mathrm{n}=207)$ & TD $(\mathrm{n}=114)$ & $\mathrm{P}$ value \\
\hline Age & $6.4 \pm 1.9$ & $6.7 \pm 1.7$ & 0.115 \\
Gender $(\mathrm{M} / \mathrm{F})$ & $178 / 29$ & $96 / 18$ & 0.666 \\
SPM & $114.4 \pm 23.4(\mathrm{n}=94)$ & $120.3 \pm 18.8(\mathrm{n}=83)$ & 0.0573 \\
ABC total score & $39.5 \pm 29.0(\mathrm{n}=117)$ & - & - \\
Irritability & $7.8 \pm 7.8$ & - & - \\
Social withdrawal & $11.2 \pm 9.0$ & - & - \\
Stereotyped behavior & $3.6 \pm 4.1$ & - & - \\
Hyperactivity & $14.1 \pm 9.8$ & - & - \\
Inappropriate & $2.8 \pm 2.6$ & - & - \\
\hline
\end{tabular}

ASD, autism spectrum disorder; TD, typically developmental; SPM Raven's Standard Progressive Matrices; ABC, Aberrant Behavior Checklist.

same time. It should be noted, however, certain limitations of the paradigms were present. For example, nonsocial stimuli exhibited two attributes as follows: repetitive stereotyped movement and geometric figure, while social stimuli exhibited another two attributes as follows: simple random movement and social human. Therefore, it is difficult to clarify the preference of children with ASD to nonsocial stimuli caused by their special interest in repetitive movement or their real preference to nonliving objects. In addition, based on previous evidence and clinical experiences, it was also speculated that visual preference for dynamic nonsocial stimuli would weaken as age was increased. Furthermore, IQ correlated negatively with the severity of ASD (23), suggesting a negative correlation between percentage of fixation time to dynamic object images (DOI) and IQ. Therefore, age and function including the IQ of subjects that may impact results should also be taken into account.

Here, to address this issue, initially, we designed and improved the paradigm on the basis of previous studies (16-18) in order to further explore the characteristics of visual preference in children with ASD. We present the following article in accordance with the SREOBE reporting checklist (available at https://dx.doi.org/10.21037/tp-21-294).

\section{Methods}

\section{Participant}

The present study enrolled children with ASD from a special education school and outpatient department of our hospital and typically developing children from kindergarten, primary school and community through advertising from September 2018 to February 2020. All ASD children were diagnosed by experienced child and adolescent psychiatrists. The inclusion criteria for the ASD group were the following: (I) meeting DSM-V criteria for ASD; (II) age range of 412 years; (III) normal vision and hearing; (IV) cooperation with eye tracking experiment. The exclusion criteria were the following: (I) subjects with other important mental illnesses, such as schizophrenia, mood disorder, anxiety disorders and tic disorders; (II) subjects with physical disease, which affected eye tracking test; (III) use of psychotropic drugs in the past one month. The subjects in the TD group did not exhibit mental diseases. The other inclusion and exclusion criteria were the same as those with the ASD group.

The number of cases during the study period determine the sample size. The case and control groups were matched for age and sex. A total of 207 children with ASD (178 males, $\mathrm{M}_{\mathrm{age}}=6.40$ years, $\mathrm{SD}_{\text {age }}=1.89$ years $)$ and $114 \mathrm{TD}$ children (96 males, $\mathrm{M}_{\text {age }}=6.74$ years, $\mathrm{SD}_{\text {age }}=1.65$ years) were enrolled. Among all subjects, 185 children (94 ASD, 91 TD) completed evaluation of intelligence. A total of 117 children with ASD were assessed with Aberrant Behavior Checklist (ABC) (Table 1).

This case-control study was conducted according to the principles of the Declaration of Helsinki (as revised in 2013) and was approved by the Ethical Committee of Peking University 6th Hospital (NO.15010000001498283), and 


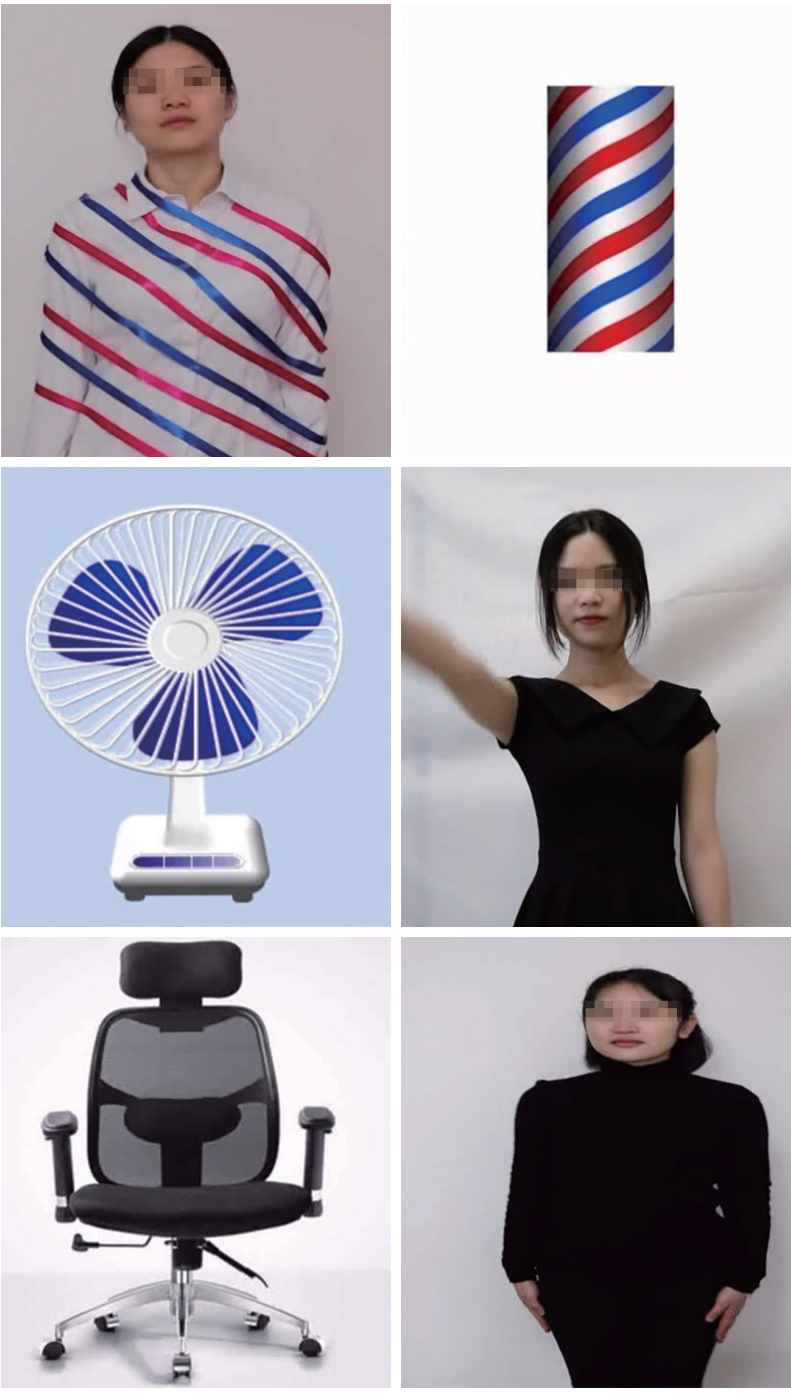

Figure 1 Sample images from the paired paradigm used in the present study. A human paired with an object with the same type and same speed of repetitive movement.

informed consent was taken from all the patients' guardians.

\section{Apparatus}

A TobiiX3-120 eye tracker and a Tobii Studio software were used to record subjects' eye tracking in response to a visual stimulus and the fixation time on it. The binocular eye tracker with infrared light sources was integrated into a 17 -in-thin film transistor monitor $(1,280 \times 1,024$ pixels). Using corneal reflection techniques, the Tobii eye tracker was set at $120 \mathrm{~Hz}$ and the $\mathrm{X}$ and $\mathrm{Y}$ coordinates of the subjects' eye position were recorded. One additional small camera was placed on the top of the eye tracking monitor to obtain the video of subject's behavior during the experiment.

\section{Paradigm}

We designed and improved the paradigm, a Dynamic Visual Preference Task, in the present study. Common objects were selected in daily life with repetitive movement, such as a revolving chair, a rotating fan and a revolving neon light, which were paired with a person with identical movement in each trial. Children with ASD and TD peers were presented with two types of stimuli on each side of a computer screen: a nonsocial stimulus-a DOI and a social stimulus-a dynamic social image (DSI). The DOI and DSI were adjusted in the same way concomitantly. A total of six pairs of videos were obtained and three pairs with social stimuli were noted on the right, whereas three pairs with social stimuli appeared on the left. Each pair of images was presented for seven sec in a continuous and random sequence (Figure 1).

\section{Procedure}

The subjects were seated at $50-80 \mathrm{~cm}$ in front of the eye tracking monitor in a room devoid of strong sunlight or light and were instructed to look at the computer screen freely. To perform calibration prior to the appearance of the stimuli of paradigm, the position of the subjects was adjusted to keep their eye tracking on the track status window. The subjects' eyes were represented by two white dots in the center of the window and the color indicator bar on the right of the window was green or yellow. Subsequently, a five-point correction was performed, which was confirmed through a Tobii Studio's automatic verification program. Only when both eyes achieved optimal mapping on five positions (smaller than 1 degree of visual angle), the calibration was deemed successful. The aforementioned process was finished and the subjects' eye tracking information was collected.

A total of 94 ASD and 83 TD children out of the entire sample size performed the Raven's Standard Progressive Matrices (SPM) test to evaluate the intelligence. The ABC questionnaire rated by parents was to assess the severity of social interaction impairment of 117 children with ASD.

\section{Data analysis}

In the present study, the following data were selected as 
independent variables for statistical analysis: total fixation duration, which included total fixation time on DOI and DSI, total fixation time on DOI or DSI, percentage of total fixation time on DOI or DSI in total fixation duration (DOI\% or DSI\%), visit duration which was indicative of average remaining time at each visit to DOI or DSI, visit count which measured the number of visits to DOI or DSI. There were 10 ASD and 2 TD without any eye movement data. We used the method of deleting tuples to deal with the missing data.

All variables accorded with normal distribution. Independent-sample $t$-test was performed to compare the independent variables between the ASD and TD group. The correlation between DOI\% or DSI\% and age, ABC scores, IQ of subjects was conducted by Pearson correlation analysis. Because whether the subjects receive school training may have an impact on visual preference, the DOI\% or DSI\% of ASD and TD children were categorized into preschool age (4-6 years) and school age (7-12 years) groups, respectively. 2 (Age group) $\times 2$ (Group) ANOVA analysis was performed to analyze the interaction between age and groups.

All statistical analyses were performed by SPSS-24 (Chicago, IL, USA). Significant differences were considered at $\mathrm{P}<0.05$ (two-sided).

\section{Results}

\section{Total fixation duration}

The total fixation duration on DOIs and DSIs were compared firstly between the ASD and TD groups. We found that the total fixation duration on DOIs and DSIs of the ASD group was obviously lower than that of the TD group $(\mathrm{t}=-7.438, \mathrm{P}<0.001)$ (Figure $2 A)$. The total fixation time on DSIs of the ASD group was significantly shorter than that of the TD group $(\mathrm{t}=-8.907, \mathrm{P}<0.001)$ (Figure $2 B$ ). No significant difference was noted in the total fixation time on DOIs between the two groups $(\mathrm{t}=-0.161, \mathrm{P}=0.872)$ (Figure 2C).

\section{Percentage of total fixation time on DOIs and DSIs in total fixation duration (DOI\% and DSI\%)}

The ASD group indicated a significant reduction of DSI\% and an increase in the DOI\% compared with the TD group $(\mathrm{t}= \pm 6.417$, both $\mathrm{P}<0.001$ ) (Figure $2 D, E)$.

$42.3 \%$ of the ASD subjects spent more than $50 \%$ of viewing time on DOI compared with only $8.7 \%$ of TD subjects. Among the subjects with ASD who preferred DOI, more than $25 \%$ spent more than $70 \%$ of their viewing time on DOI, with several subjects exceeding $80 \%$ on DOI.

\section{Visit duration and visit count}

The visit duration on DOIs was longer in the ASD group than that in the TD group $(\mathrm{t}=5.757, \mathrm{P}<0.001)$ (Figure $2 F)$. No significant difference was noted in the visit duration on DSIs between the two groups $(\mathrm{t}=-2.017, \mathrm{P}=0.045)$ (Figure $2 G$ ). The ASD group was lower than that of the TD group both on DOI $(\mathrm{t}=-4.201, \mathrm{P}<0.001)$ and DSI $(\mathrm{t}=-5.544$, $\mathrm{P}<0.001$ ) in the visit count (see Figure $2 H, I$ ).

\section{Eye tracking characteristics and age}

\section{Association between DOI\% or DSI\% and age}

Within the ASD group, DOI\% correlated negatively with age, whereas DSI\% correlated positively with age $(\mathrm{R}= \pm 0.225$, both $\mathrm{P}=0.001)$. No correlation was noted between DOI\% or DSI\% and age in the TD group $(\mathrm{R}= \pm 0.06, \mathrm{P}=0.528)$ (Figure 3).

\section{Relationship between DOI\% or DSI\% and IQ}

No difference was noted in the IQ between the two groups. The ASD group exhibited a significantly increased DOI\% and a significantly decreased DSI\% than those of the TD group. No association was noted between DOI\% or DSI\% and IQ in the ASD group $(\mathrm{R}=0.081, \mathrm{P}=0.435)$.

\section{Association between DOI\% or DSI\% and ASD symptoms}

The correlations of DOI\% or DSI\% with the scores of ABCs were analyzed. Among the five subscales, DOI\% correlated positively with the score of social withdrawal/ stagnation subscale when age was controlled $(r=0.227$, $\mathrm{P}=0.014)$. By contrast, DSI\% was negatively associated with the score of the same subscale $(\mathrm{r}=-0.227, \mathrm{P}=0.014)$. No correlations were found between DOI\% or DSI\% and the scores of other subscales (Table 2).

\section{Discussion}

Recently, an accumulated number of studies have demonstrated a visual preference of children with ASD. However, the results are not completely consistent. To further explore the characteristics of visual preference 

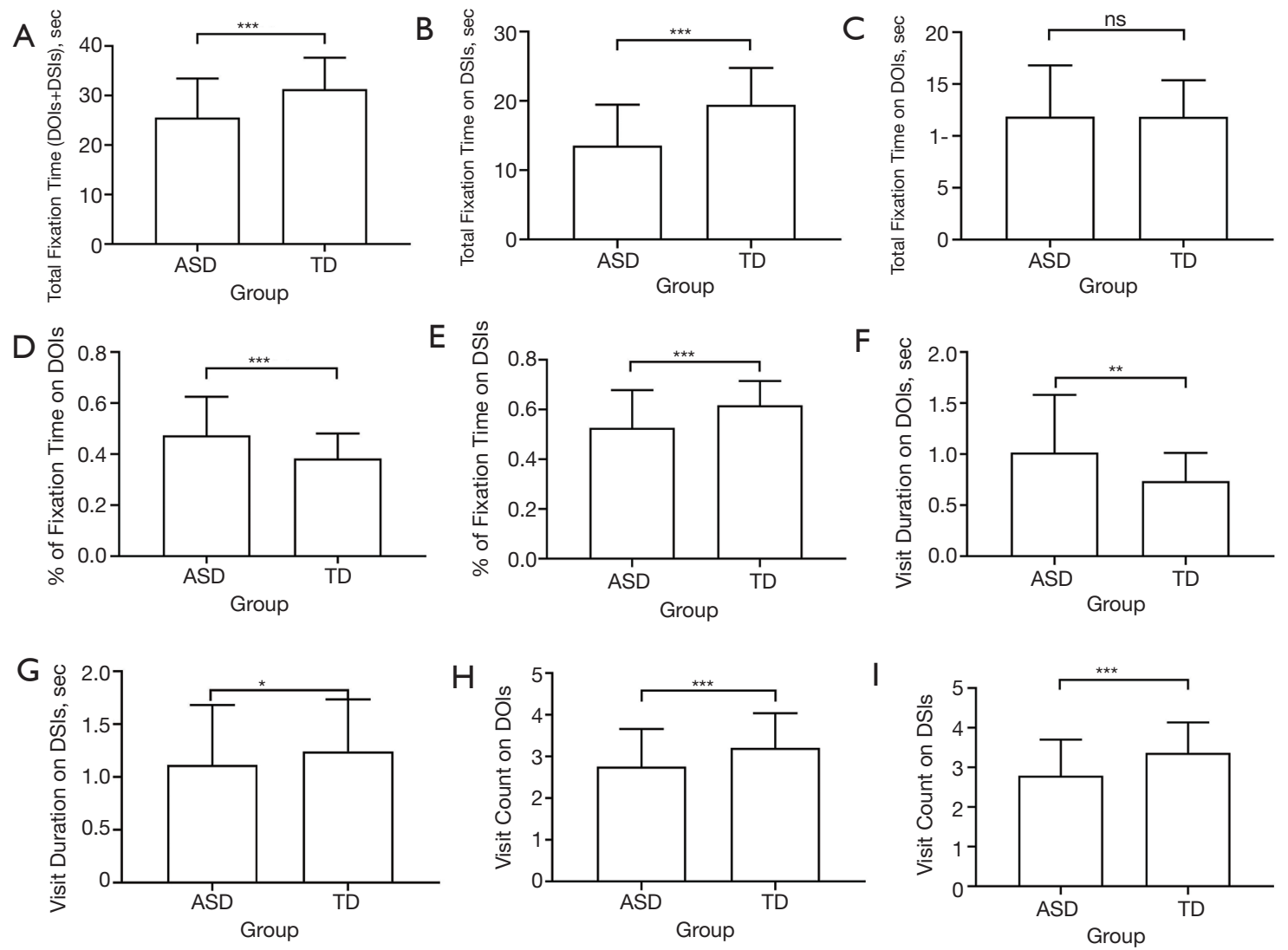

Figure 2 The comparison of visual preference between ASD and TD children. (A) Total fixation time on DOIs and DSIs between the two groups; (B) total fixation time on DSIs between the two groups; (C) total fixation time on DOIs between the two groups; (D) percentage of fixation time on DOI between the two groups; (E) percentage of fixation time on DSI between the two groups; (F) visit duration on DSIs between the two group; $(\mathrm{G})$ visit duration on DOIs between the two group; (H) visit count on DOIs between the two group; (I) visit count on DOIs between the two group. ${ }^{* * *} \mathrm{P}<0.001,{ }^{* *} \mathrm{P}<0.01,{ }^{*} \mathrm{P}<0.05$, DOI, dynamic object image; DSI, dynamic social image.

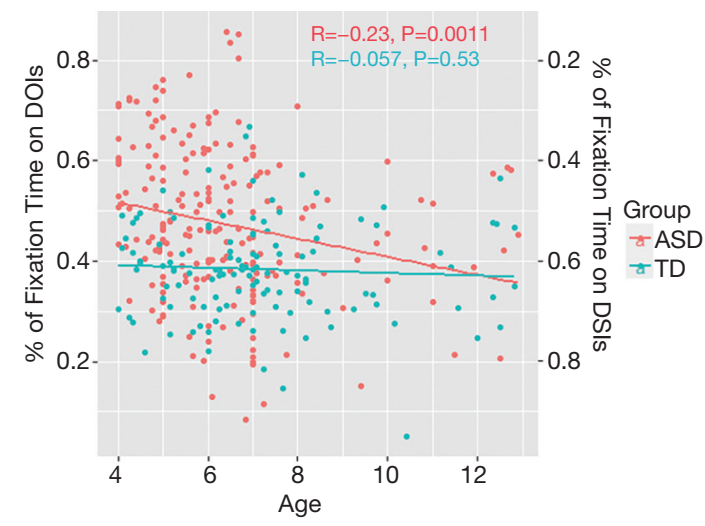

Figure 3 The relationship between DOI\% or DSI\% and ages in ASD and TD group. DOI, dynamic object image; ASD, autism spectrum disorder; TD, typically developmental; DSI, dynamic social image.
Table 2 Correlation of DOI\% or DSI\% with the score of ABCs in ASD group

\begin{tabular}{lc}
\hline ABC terms & ASD group, DOI\% (DSI\%) \\
\hline ABC total & $0.141(-0.141)$ \\
Irritability & $0.056(-0.056)$ \\
Social withdrawal & $0.237^{\star \star}\left(-0.237^{\star *}\right)$ \\
Stereotype & $0.122(-0.122)$ \\
Hyperactivity & $0.123(-0.123)$ \\
Inappropriate & $-0.008(0.008)$
\end{tabular}

${ }^{* *} P \leq 0.01$. DOI, dynamic object image; DSI, dynamic social image; ABC, Aberrant Behavior Checklist; ASD, autism spectrum disorder. 
of children with ASD, a visual preference paradigm was designed based on previous studies. By controlling repetitive movement of the two types of stimuli, the visual preference of the children with ASD for social and nonsocial stimuli could be accurately studied.

In the current study, 207 children with ASD and 125 TD children with an age range of 4-12 years were studied. The results indicated that (I) the total fixation time of ASD children on social and nonsocial stimuli was significantly lower than that of the TD children; (II) the total fixation time of the ASD group on DSI was significantly lower than that of the TD group, while no significant difference was noted between the two groups in the total fixation time on DOI; (III) the children with ASD exhibited significantly lower DSI\% and higher DOI\% compared to those noted in the TD group; (IV) the ASD showed more mean duration of each visit on the DOI and less mean duration of each visit on the DSI compared to the TD; (V) the ASD group exhibited much fewer visit counts than the TD group regardless of whether DOI or DSI were assessed.

Due to the visual preference paradigm of the present study, which controlled the confounding effect of repetitive movement, the aforementioned results could reflect the features of the visual preference of children with ASD in a more efficient way. The results suggested that children with ASD spent less total fixation time on the stimuli (DSI + DOI) than those of the TD group. Since no significant difference was noted in the total fixation time of DOI between the two groups and based on the fact that the ASD group spent less fixation time on DSI than the TD group, the decreased total fixation duration on stimuli (DSI + DOI) was mainly due to the significant reduction of fixation time on DSI. In addition, the overall time spent on DSI was significantly reduced and DSI\% was significantly lower than that of the TD group, which indicated that children with ASD paid less attention to social stimuli than those of the TD group. Although no significant differences were noted in the absolute fixation time on nonsocial stimuli between the two groups, the proportion of fixation time on nonsocial stimuli in total fixation duration was apparently increased compared with that of the TD group, indicating that children with ASD paid more attention to nonsocial stimuli than children with TD. These results are consistent with those of previous studies, which also showed that compared with TD children, children with ASD were more inclined to look at non-living objects over humans $(16,21,24,25)$. In addition, the present study demonstrated the average visit time on social stimuli and the number of visits was significantly reduced compared to the TD group. Although the number of visits to nonsocial stimuli was apparently decreased, the average visit time on nonsocial stimuli was longer than that of the TD group. Since no significant difference was noted in the total fixation time on DOI between the two groups, the results further suggested that the children with ASD exhibited reduced interests in social stimuli and paid less attention to them, while they had more interests in nonsocial stimuli and preferred to watch them. Once the gaze of ASD children eyes shifted to nonsocial stimuli, they remained longer on that state, which was identified as "sticky attention". This finding was in line with the results of a previous study (26). It is not clear why ASD children were less interested in social stimulation and more interested in nonsocial stimulation. A possible hypothesis could be based on social information being more unpredictable for ASD compared with TD. The visual preference to nonsocial objects may be a way to comfort them from the unpredictable social world to a predictable world. However, insufficient evidence is present to support this hypothesis and further research is required to explore the possible psychological and brain mechanism in the future.

The present study further explored the association between visual preference and clinical symptoms of ASD by analyzing the correlation between DSI\% or DOI\% and the scores of ABCs. The results indicated that in children with ASD, DSI\% correlated negatively with the score of social withdrawal subscale of $\mathrm{ABC}$, whereas DOI\% exhibited a significant positive correlation with the score of the same subscale of $\mathrm{ABC}$. These results suggested that the visual preference of children with ASD was relevant to their social deficits. The more attention they paid to nonsocial stimuli and the less attention to social stimuli resulted in more serious social interaction defects and vice versa. These results were in accordance with previous studies demonstrating that the social deficit of children with ASD was significantly associated with their fixation time on social information. The more severe the social impairment, the less attention was paid to social information. In the current study, no significant correlation was found between visual preference and scores of other $\mathrm{ABC}$ subscales (irritation, stereotyped behavior, hyperactivity, inappropriate language) in children with ASD, which further suggested that the fixation preference to social and nonsocial stimuli was mainly associated with social interaction impairments in children with ASD. However, it has been shown that children with ASD prefer repetitive movement rather than 
random movement and that the preference to repetitive movement correlates positively with the score of restricted interests subscale of Repetitive Behavior Scale-Revised (RBS-R) (27). The purpose and paradigm of that study were different from that of the current study. In that study, the visual preference of ASD children to repetitive or random movement was explored by the paradigm of matching repetitive and random motion to objects. Since ASD children preferred repetitive movement, identical repetitive movement was provided to both social and nonsocial stimuli in the present study, so as to eliminate the confounding effect of this parameter to our results and clarify the visual preference of ASD children to social and nonsocial stimuli.

In the current study, a significant correlation was noted in the ASD group between the parameters DOI\% or DSI\% and age. As the age increased, the children with ASD paid more attention to social stimuli and less attention to nonsocial stimuli, which suggested that social defects of children with ASD were improved as age was increased. This result is accordant with the results reported in previous systematic reviews and meta-analyses, which indicated that the scores of social interaction defects (such as eye contact, joint attention, social overtures and others) in ADOS were decreased as age increased in children with ASD (28). A previous meta-analysis (29) further demonstrated that the total score of ADOS was decreased in children receiving specific intervention. Dawson et al. found that cortical activation in response to faces vs. objects was normalized in children after the two year's Early Start Denver Model intervention (30). In the present study, all children with ASD received education intervention and therefore their social interaction behavior and preference to social stimuli improved with the increase of age.

Unfortunately, contrary to this hypothesis, we did not find a correlation between fixation preference to social or nonsocial stimuli and IQ in children with ASD. The reason for this may be related to the feature of SPM, which measures IQ only through distinguishing and reasoning figures and cannot reflect the individual's intelligence comprehensively. So in future studies, other intelligence tests (such as Wechsler Intelligence Scale) should be used, which can evaluate intelligence comprehensively and further explore the association between visual preference and IQ in children with ASD.

In the present study, the visual preference paradigm was improved to reduce the bias caused by different movement of social and nonsocial stimuli and the samples size was the largest reported so far compared with similar studies. However, the current study contains certain limitations. For example, the diagnosis of ASD was carried out by experienced child psychiatrists based on DSM-V diagnostic criteria without Autism Diagnostic Interview (ADI) or Autism Diagnostic Observation Schedule (ADOS) assessment. The control group only included normal developing children and subjects under 4 years old were not included. Moreover, no follow-up was performed on the subjects that participated in the study. Therefore, in future studies, ADI or ADOS will be included in the process of diagnosis of ASD and the study subjects will be expanded to include children with other diagnostic criteria and subjects under 4 years of age. The subjects should be followed up regularly, so as to explore the visual preference feature in children with ASD in more detail.

In conclusion, children with ASD preferred to look at nonsocial stimuli rather than social stimuli. The preference degree to social stimuli was negatively associated with the severity of social interaction impairment of ASD and was improved when the age of the ASD children was increased.

\section{Acknowledgments}

We are grateful to all the participants and their families, as well as all the staff contributed to this study. The language of our paper is edited by the qualified scientific editors at MedSci.

Funding: National Key R\&D Program of China (No. 2017YFC1309900), Beijing Municipal Science \& Technology Commission (Nos. Z171100001017088), National Natural Science Foundation of China (No. 82171508, 81571339).

\section{Footnote}

Reporting Checklist: The authors have completed the STROBE reporting checklist. Available at https://dx.doi. org/10.21037/tp-21-294

Data Sharing Statement: Available at https://dx.doi. org/10.21037/tp-21-294

Conflicts of Interest: All authors have completed the ICMJE uniform disclosure form (available at https://dx.doi. org/10.21037/tp-21-294). The authors have no conflicts of interest to declare.

Ethical Statement: The authors are accountable for all 
aspects of the work in ensuring that questions related to the accuracy or integrity of any part of the work are appropriately investigated and resolved. The study was conducted in accordance with the Declaration of Helsinki (as revised in 2013). The study was approved by the Ethical Committee of Peking University 6th Hospital (NO.15010000001498283) and informed consent was taken from all the patients' guardians.

Open Access Statement: This is an Open Access article distributed in accordance with the Creative Commons Attribution-NonCommercial-NoDerivs 4.0 International License (CC BY-NC-ND 4.0), which permits the noncommercial replication and distribution of the article with the strict proviso that no changes or edits are made and the original work is properly cited (including links to both the formal publication through the relevant DOI and the license). See: https://creativecommons.org/licenses/by-nc-nd/4.0/.

\section{References}

1. von dem Hagen EA, Stoyanova RS, Baron-Cohen S, et al. Reduced functional connectivity within and between 'social' resting state networks in autism spectrum conditions. Soc Cogn Affect Neurosci 2013;8:694-701.

2. Möricke E, Buitelaar JK, Rommelse NNJ. Do We Need Multiple Informants When Assessing Autistic Traits? The Degree of Report Bias on Offspring, Self, and Spouse Ratings. J Autism Dev Disord 2016;46:164-75.

3. Moore A, Wozniak M, Yousef A, et al. The geometric preference subtype in ASD: identifying a consistent, earlyemerging phenomenon through eye tracking. Mol Autism 2018;9:19.

4. Chawarska K, Macari S, Shic F. Decreased spontaneous attention to social scenes in 6-month-old infants later diagnosed with autism spectrum disorders. Biol Psychiatry 2013;74:195-203.

5. Chawarska K, Ye S, Shic F, et al. Multilevel Differences in Spontaneous Social Attention in Toddlers With Autism Spectrum Disorder. Child Dev 2016;87:543-57.

6. Kirchner JC, Hatri A, Heekeren HR, et al. Autistic symptomatology, face processing abilities, and eye fixation patterns. J Autism Dev Disord 2011;41:158-67.

7. Klin A, Jones W, Schultz R, et al. Visual fixation patterns during viewing of naturalistic social situations as predictors of social competence in individuals with autism. Arch Gen Psychiatry 2002;59:809-16.

8. Chawarska K, Macari S, Shic F. Context modulates attention to social scenes in toddlers with autism. J Child Psychol Psychiatry 2012;53:903-13.

9. Frazier TW, Strauss M, Klingemier EW, et al. A Meta-Analysis of Gaze Differences to Social and Nonsocial Information Between Individuals With and Without Autism. J Am Acad Child Adolesc Psychiatry 2017;56:546-55.

10. Tanaka JW, Sung A. The "Eye Avoidance" Hypothesis of Autism Face Processing. J Autism Dev Disord 2016;46:1538-52.

11. Jones W, Carr K, Klin A. Absence of preferential looking to the eyes of approaching adults predicts level of social disability in 2-year-old toddlers with autism spectrum disorder. Arch Gen Psychiatry 2008;65:946-54.

12. Shic F, Bradshaw J, Klin A, et al. Limited activity monitoring in toddlers with autism spectrum disorder. Brain Res 2011;1380:246-54.

13. Klin A, Lin DJ, Gorrindo P, et al. Two-year-olds with autism orient to non-social contingencies rather than biological motion. Nature 2009;459:257-61.

14. Bedford R, Elsabbagh M, Gliga T, et al. Precursors to social and communication difficulties in infants at-risk for autism: gaze following and attentional engagement. J Autism Dev Disord 2012;42:2208-18.

15. Vivanti G, Fanning PAJ, Hocking DR, et al. Social Attention, Joint Attention and Sustained Attention in Autism Spectrum Disorder and Williams Syndrome: Convergences and Divergences. J Autism Dev Disord 2017;47:1866-77.

16. Pierce K, Conant D, Hazin R, et al. Preference for geometric patterns early in life as a risk factor for autism. Arch Gen Psychiatry 2011;68:101-9.

17. Pierce K, Marinero S, Hazin R, et al. Eye Tracking Reveals Abnormal Visual Preference for Geometric Images as an Early Biomarker of an Autism Spectrum Disorder Subtype Associated With Increased Symptom Severity. Biol Psychiatry 2016;79:657-66.

18. Sasson NJ, Touchstone EW. Visual attention to competing social and object images by preschool children with autism spectrum disorder. J Autism Dev Disord 2014;44:584-92.

19. Bodfish JW, Symons FJ, Parker DE, et al. Varieties of repetitive behavior in autism: comparisons to mental retardation. J Autism Dev Disord 2000;30:237-43.

20. Happé F, Frith U. The weak coherence account: detailfocused cognitive style in autism spectrum disorders. J Autism Dev Disord 2006;36:5-25.

21. Kou J, Le J, Fu M, et al. Comparison of three different eye-tracking tasks for distinguishing autistic from typically 
developing children and autistic symptom severity. Autism Res 2019;12:1529-40.

22. Fischer J, Koldewyn K, Jiang YV, et al. Unimpaired Attentional Disengagement and Social Orienting in Children with Autism. Clin Psychol Sci 2014;2:214-23.

23. Bedford SA, Park MTM, Devenyi GA, et al. Largescale analyses of the relationship between sex, age and intelligence quotient heterogeneity and cortical morphometry in autism spectrum disorder. Mol Psychiatry 2020;25:614-28.

24. Fujioka T, Inohara K, Okamoto Y, et al. Gazefinder as a clinical supplementary tool for discriminating between autism spectrum disorder and typical development in male adolescents and adults. Mol Autism 2016;7:19.

25. Shaffer RC, Pedapati EV, Shic F, et al. Brief Report: Diminished Gaze Preference for Dynamic Social Interaction Scenes in Youth with Autism Spectrum Disorders. J Autism Dev Disord 2017;47:506-13.

26. Fischer J, Smith H, Martinez-Pedraza F, et al. Unimpaired

Cite this article as: Gong $\mathrm{X}, \mathrm{Li} \mathrm{X}$, Wang Q, Hoi SP, Yin T, Zhao L, Meng F, Luo X, Liu J. Comparing visual preferences between autism spectrum disorder (ASD) and normal children to explore the characteristics of visual preference of ASD children by improved visual preference paradigm: a case-control study. Transl Pediatr 2021;10(8):2006-2015. doi: 10.21037/tp21-294 attentional disengagement in toddlers with autism spectrum disorder. Dev Sci 2016;19:1095-103.

27. Wang Q, Hu Y, Shi D, et al. Children with Autism Spectrum Disorder Prefer Looking at Repetitive Movements in a Preferential Looking Paradigm. J Autism Dev Disord 2018;48:2821-31.

28. Bieleninik Ł, Posserud MB, Geretsegger M, et al. Tracing the temporal stability of autism spectrum diagnosis and severity as measured by the Autism Diagnostic Observation Schedule: A systematic review and meta-analysis. PLoS One 2017;12:e0183160.

29. Virués-Ortega J. Applied behavior analytic intervention for autism in early childhood: meta-analysis, meta-regression and dose-response meta-analysis of multiple outcomes. Clin Psychol Rev 2010;30:387-99.

30. Dawson G, Jones EJ, Merkle K, et al. Early behavioral intervention is associated with normalized brain activity in young children with autism. J Am Acad Child Adolesc Psychiatry 2012;51:1150-59. 